\title{
Antibiotic management of urinary tract infection in elderly patients in primary care and its association with bloodstream infections and all cause mortality: population based cohort study
}

\author{
Myriam Gharbi, ${ }^{1,2}$ Joseph H Drysdale, ${ }^{3}$ Hannah Lishman, ${ }^{1,2}$ Rosalind Goudie, ${ }^{1,2,4}$ \\ Mariam Molokhia, ${ }^{5}$ Alan P Johnson, ${ }^{1,6}$ Alison H Holmes, ${ }^{1}$ Paul Aylin ${ }^{1,2}$
}

${ }^{1}$ NIHR Health Protection

Research Unit, Healthcare Associated Infections and Antimicrobial Resistance, Imperial College London, London, UK

${ }^{2}$ Department of Primary Care and Public Health, Imperial College London, London, UK ${ }^{3}$ Medical School, St George's University of London, London, UK

${ }^{4}$ Nuffield Department of Population Health, University of Oxford, Oxford, UK

${ }^{5}$ Department of Primary Care and Public Health Sciences, King's College, London, UK

${ }^{6}$ Healthcare-Associated Infections and Antimicrobial Resistance Division, National Infection Service, Public Health England, London, UK Correspondence to: P Aylin p.aylin@imperial.ac.uk (ORCID 0000-0003-4589-1743) Additional material is published online only. To view please visit the journal online.

Cite this as: $B M / 2019 ; 364: 1525$ http://dx.doi.org/10.1136/bmj.l525

Accepted: 16 January 2019

\section{ABSTRACT}

OBJECTIVE

To evaluate the association between antibiotic treatment for urinary tract infection (UTI) and severe adverse outcomes in elderly patients in primary care.

DESIGN

Retrospective population based cohort study.

\section{SETTING}

Clinical Practice Research Datalink (2007-15) primary care records linked to hospital episode statistics and death records in England.

\section{PARTICIPANTS}

157264 adults aged 65 years or older presenting to a general practitioner with at least one diagnosis of suspected or confirmed lower UTI from November 2007 to May 2015.

\section{MAIN OUTCOME MEASURES}

Bloodstream infection, hospital admission, and all cause mortality within 60 days after the index UTI diagnosis.

RESULTS

Among 312896 UTI episodes (157 264 unique patients), $7.2 \%(n=22534)$ did not have a record of antibiotics being prescribed and $6.2 \%(n=19292)$ showed a delay in antibiotic prescribing. 1539 episodes of bloodstream infection $(0.5 \%)$ were recorded within 60 days after the initial UTI. The rate of bloodstream infection was significantly higher among those patients not prescribed an antibiotic (2.9\%; $n=647)$ and those recorded as revisiting the general practitioner within seven days of the initial consultation for an antibiotic prescription compared with those given a prescription for an antibiotic at the initial consultation ( $2.2 \% \vee 0.2 \%$; $\mathrm{P}=0.001)$. After

\section{WHAT IS ALREADY KNOWN ON THIS TOPIC}

About half of Escherichia coli bloodstream infections are caused by an underlying urinary tract infection (UTI), with higher risk seen in elderly people

While "no antibiotic" or "delayed or deferred antibiotic" treatment is often not associated with severe adverse outcomes for some self limiting illnesses (eg, upper respiratory tract infections), a slight increase in symptom duration and complication rate have been reported for UTI in young women

The generalisability of these studies, however, is limited because of sample size and study population demographics

\section{WHAT THIS STUDY ADDS}

After adjustment for key covariates, no antibiotics and deferred antibiotic

approaches for the management of UTI in older adults in primary care appears to be associated with a significant increased risk of bloodstream infection and all cause mortality compared with an immediate antibiotics approach adjustment for covariates, patients were significantly more likely to experience a bloodstream infection in the deferred antibiotics group (adjusted odds ratio $7.12,95 \%$ confidence interval 6.22 to 8.14 ) and no antibiotics group (8.08, 7.12 to 9.16$)$ compared with the immediate antibiotics group. The number needed to harm (NNH) for occurrence of bloodstream infection was lower (greater risk) for the no antibiotics group $(\mathrm{NNH}=37)$ than for the deferred antibiotics group $(\mathrm{NNH}=51)$ compared with the immediate antibiotics group. The rate of hospital admissions was about double among cases with no antibiotics (27.0\%) and deferred antibiotics (26.8\%) compared with those prescribed immediate antibiotics $(14.8 \% ; \mathrm{P}=0.001)$. The risk of all cause mortality was significantly higher with deferred antibiotics and no antibiotics than with immediate antibiotics at any time during the 60 days follow-up (adjusted hazard ratio 1.16, 95\% confidence interval 1.06 to 1.27 and $2.18,2.04$ to 2.33, respectively). Men older than 85 years were particularly at risk for both bloodstream infection and 60 day all cause mortality.

\section{CONCLUSIONS}

In elderly patients with a diagnosis of UTI in primary care, no antibiotics and deferred antibiotics were associated with a significant increase in bloodstream infection and all cause mortality compared with immediate antibiotics. In the context of an increase of Escherichia coli bloodstream infections in England, early initiation of recommended first line antibiotics for UTI in the older population is advocated.

\section{Introduction}

Urinary tract infection (UTI) is the most common bacterial infection in the older patient population, and Escherichia coli is the most common uropathogen in community dwelling people older than 65 years. ${ }^{1}$ The spectrum of UTI ranges from a mild self limiting illness to severe sepsis, with a mortality rate of 20$40 \%$. The incidence of sepsis and its associated mortality increases disproportionately with age, and UTI in men is more likely to be severe. ${ }^{2-4}$ Both sexes develop UTI in old age, with a female to male ratio of 2:1 in patients older than 70 years, compared with the overwhelming UTI susceptibility of females in younger populations, with a 50:1 ratio. ${ }^{5}$ The diagnosis of UTI in older patients can be problematic, as these patients are less likely to present with a typical clinical history and localised urinary symptoms compared with younger patients. $^{6}$ The rising incidence of asymptomatic bacteriuria in older adults is also contributing to further diagnostic difficulty $(>20 \%$ of women aged 
$\geq 65$ years compared with $<5 \%$ in younger women), which results in probable over-diagnosis of UTI and unnecessary treatment. ${ }^{6-8}$

UTI is the second most common diagnosis for which empirical antibiotics are prescribed in both primary and secondary care, with more than 50\% of the antibiotics prescribed for a suspected UTI in older adults being considered unnecessary. ${ }^{9-11}$ With the spread of antibiotic resistance and its increasing threat to public health (about $30 \%$ of urinary isolates of $E$ coli are now resistant to trimethoprim), national guidelines and antimicrobial stewardship programmes have been proposed to combat these challenges. ${ }^{12-16}$ NHS England, for example, released the Quality Premium to incentivise Clinical Commissioning Groups to reduce antibiotic use in primary care. ${ }^{17}$ As a result of these new initiatives, a substantial decrease in antibiotic use has been reported for the first time in England across the whole healthcare system between 2013 and 2017. ${ }^{16-19}$ A recent study has also shown a decrease in prescribing of broad spectrum antibiotics for UTI in older people in primary care between 2004 and $2014 .^{20}$ In the meantime, however, increases in the incidence of Gram negative bloodstream infections have been reported, which has led the UK government to announce a plan to reduce healthcare associated Gram negative bloodstream infections in England by $50 \%$ by March 2021. ${ }^{16}$

As the pattern of antibiotic use changes in the context of antimicrobial resistance, it is now more important than ever to assess the management and outcome of UTIs. Clostridium difficile in elderly people has also been one of the drivers for scrutiny of unnecessary antibiotic use in this population. A decline in antibiotic use may, however, harm vulnerable older populations who are already more likely to develop UTI related complications and bloodstream infection. More evidence is needed about the initial treatment of UTI in primary care, including an assessment of prescribing approaches involving no antibiotics, deferred antibiotics, or immediate antibiotics, and the subsequent clinical outcome. We linked primary care data in England with hospital admissions and mortality data at a patient level, allowing a pragmatic approach to assessing the impact of standard care in the community for a large cohort of older patients with confirmed or suspected UTI on adverse events, including hospital admission, bloodstream infection, and death.

\section{Methods}

We conducted a retrospective population based cohort study in England on patients attending National Health Service general practices submitting data to the UK based Clinical Practice Research Datalink (CPRD) between November 2007 and June 2015.

\section{Data source}

Anonymous medical patient records were extracted from CPRD, the world's largest primary care electronic health database containing information on a representative national sample. About $7 \%$ of English
NHS general practices across the country contribute data to this database. ${ }^{21}$ CPRD has been extensively used and validated for pharmacoepidemiological research.

The CPRD database contains a wide ranging set of information, which includes patient sociodemographics, medical diagnoses using the READ classification system, outpatient prescriptions, physiological and laboratory investigations, health behaviours, and referrals to secondary care. ${ }^{22}$ More than $50 \%$ of the practices registered with CPRD have agreed to linkage of their records with the corresponding patient hospital records from the hospital episode statistics database, which contains information on all hospital admissions, together with information about the causes of each episode of inpatient care using ICD-10 (international classification of diseases, 10th revision) for the coding of diagnosis, type of admission, procedure performed, length of stay, and discharge status (https://digital.nhs.uk/data-andinformation/data-tools-and-services/data-services/ hospital-episode-statistics).

We also linked the patients' primary care data to the death registration data from the Office for National Statistics, which contain date and causes of death, and to the 2010 English index of multiple deprivation data, which contain small area level measures of relative deprivation. For the latter, we obtained a proxy for sociodemographic and socioeconomic status across numerous domains, including housing, employment, income, access to services, education and skills, crime, and living environment, using practice postcode for data linkage (www.cprd.com/dataAccess/linkeddata.asp).

\section{Population}

All patients aged 65 years or older presenting to a general practitioner (GP) with at least one diagnosis of suspected or confirmed lower UTI (recorded using a READ code indicating a clinical test or referral event) in the CPRD database, were included in the study (see supplementary table S1). Patients were excluded if they presented with asymptomatic bacteriuria or had missing data for sex. They were also excluded if they had a diagnosis of a complicated UTI, were admitted to hospital, or died on the same day as their initial UTI diagnosis.

All study participants were registered with a practice for at least 12 continuous months before their first UTI consultation (defined as the index UTI) to capture potential comorbidities and medical history.

To distinguish distinct episodes of UTI for the same patient, we used a period of 90 days, comprising 30 days before diagnosis and 60 day follow-up after the index UTI. We considered all the GP consultations within 60 days of the initial UTI diagnosis as being related to the same UTI episode. To identify any relevant medical history for a new episode we used a 30 day buffer period before the index date of the second UTI episode (fig 1).

To ensure data quality we only included practices classified as up to standard (continuous high quality 


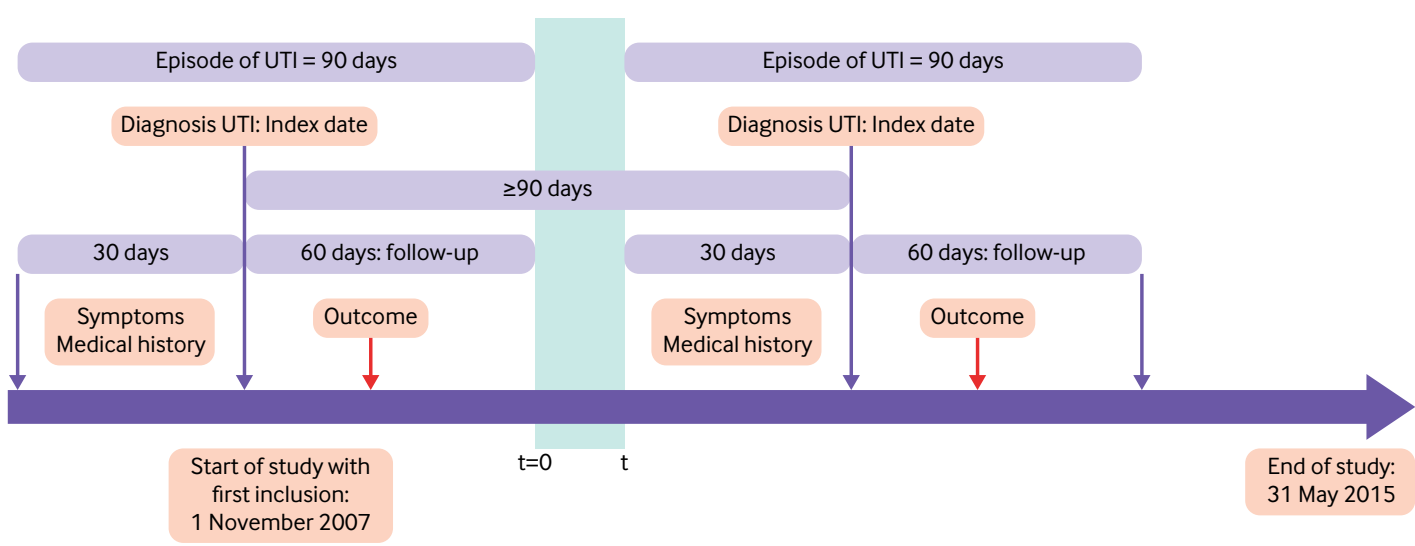

Fig 1 | Timeline of study and criteria for differentiating independent episodes of urinary tract infection (UTI)

data acceptable for use in research) a year before the start of the study period. Similarly, we only included patient data if an acceptable registration status for use in research, including contiguous follow-up and valid data recording as defined by CPRD, was present at the time of the recruitment and during the follow-up period. ${ }^{23}$ As part of the data management process, we considered UTI observations with the same consultation date to be duplicates. All additional information present in different rows in the database on the same date were grouped together under a same GP consultation.

We further excluded patients for whom primary care medical records were not eligible for data linkage with the hospital episode statistics records and patients without a 60 day follow-up period after the index UTI consultation.

\section{Exposure}

The main exposures were antibiotic prescribing practices after the initial diagnosis of UTI in primary care, defined as immediate antibiotics (patients prescribed an antibiotic during first UTI visit or on same day), deferred antibiotics (patients prescribed an antibiotic within seven days to allow for the natural resolution of the disease, ${ }^{24}$ but not on the day of the initial UTI diagnosis and in the absence of complication or hospital admission, or both), and no antibiotics (patients with no record of having been prescribed an antibiotic by the GP within seven days after the UTI diagnosis or if a complication occurred before antibiotics were prescribed). The name of the antibiotic and duration of treatment were also collected.

The deferred antibiotics group should capture the post-dated prescriptions given to patients on the index date or left behind at the reception to collect on a later date, or the prescriptions for patients who were asked to return if symptoms did not improve.

\section{Outcome}

The primary outcomes of interest were bloodstream infection within 60 days after the initial diagnosis, captured in both hospital episode statistics and CPRD (our definition of bloodstream infection included Read codes and ICD-10 codes related to sepsis, septicaemia, and bacteraemia; see supplementary tables S1 and S2) and all cause mortality within 60 days after the initial UTI diagnosis. The secondary outcomes of interest were hospital admission within 60 days after a UTI diagnosis, length of stay for patients admitted to hospital during a UTI episode, and type of care pathway experienced by the patient.

We classified the patients into three different types of care pathways: single primary care consultation for a UTI without hospital admission or complication related to the UTI within 60 days of the initial diagnosis, multiple primary care consultations for the same UTI episode without hospital admission within 60 days of the initial diagnosis, and single or multiple primary care consultations for the same UTI episode with a hospital admission, regardless of the reason, within 60 days of the UTI diagnosis, and including any UTI related complications (see supplementary tables S1 and S2), bloodstream infection, or death.

\section{Covariates}

A set of covariates was described and used in the models to adjust for potential sources of confounding. Covariates included age (defined as a categorical variable: $65-74,75-84$, and $\geq 85$ years), sex, grouped regions (defined as a categorical variable: North of England and Yorkshire, Midlands and east of England, south of England, and London), area level deprivation (index of multiple deprivation) divided into fifths (first fifth being the least deprived and last fifth the most deprived), year of consultations/ diagnoses (financial years from May to April to account for changes in NHS England quality premium guidance), ${ }^{17}$ Charlson comorbidity score (scale from 0 to 12 , with higher scores indicating increased risk of death within a year) (see supplementary table S3), ${ }^{25}$ immunosuppression, smoking status, medical history 30 days before the index UTI (indwelling urethral catheter, hospital admission with a discharge date within the 30 days before the index case, antibiotic exposure including short course or prophylactic treatment, presenting symptoms potentially related to UTI), and a history of recurrent UTIs. 
We defined a recurrent UTI as the presence of a Read code for recurrent UTI or prophylactic treatment for UTI (trimethoprim or nitrofurantoin prescribed for $\geq 28$ days) or two or more UTIs within 12 months. Although recurrent UTI is usually defined as two or more UTIs within six months or three or more within 12 months, ${ }^{26}$ we adapted this definition to account for the 90 day period between each index case to allow for distinct UTI episodes.

When data were missing on binary covariates, we classified these as absence of the condition.

\section{Statistical analyses}

We first compared patient characteristics and other covariates with different uses of antibiotics. The variables of interest were described using standard measures of central tendency and variability-that is, means and standard deviations for continuous variables and counts and percentages for categorical variables. Comparisons between groups were performed using a range of tests: $\chi^{2}$ and Fisher's exact tests for categorical variables, analysis of variance, and the Kruskal-Wallis for continuous variables. We also compared the rates of bloodstream infection, hospital admission, and all cause mortality as well as the average length of stay for patients admitted to hospital between the three antibiotic groups. The numbers needed to harm (NNH) related to both bloodstream infection and death within 60 days were then calculated. This measure indicated the average number of patients needed to be exposed to no antibiotics and deferred antibiotics to cause harm in an average of one patient who would not otherwise have been harmed if treated with immediate antibiotics.

Differences in the proportion of cases experiencing one of the three care pathways were stratified by antibiotic use, age, and sex and were compared using the $\chi^{2}$ test.

The secondary analysis evaluated the predictors of bloodstream infection and death within 60 days after the index UTI. We first constructed the Kaplan-Meier curves for time to death within 60 days and then stratified by antibiotic use and the use of the two first line antibiotics (trimethoprim and nitrofurantoin) at the first visit to the GP. After ensuring the proportional hazard assumptions were met, we compared the curves using the log rank test to assess significance. The main predictor analysed for this study was antibiotic use.

To assess the associations between antibiotic use and bloodstream infection, we performed a multivariable logistic regression analysis, whereas to assess the association between antibiotic use and all cause mortality within 60 days after a UTI diagnosis we used a multivariable Cox regression analysis. The distinct episodes of UTI within the same patient are likely to be correlated with each other, which may affect the apparent relation between antibiotic use and outcome. Not accounting for intracluster correlation and assuming independence between episodes might lead to smaller standard errors and thus narrower confidence intervals for the variable estimates.
Therefore we used the robust standard error approach in both logistic and Cox regression models to derive standard errors that allow for the clustering.

A sensitivity analysis was subsequently undertaken to assess the risks of outcomes selected with use of any antibiotic for any duration. We restricted the sensitivity analyses to antibiotic treatment with durations of fewer than 21 days and 28 days to target only curative treatment (as longer duration of antibiotic use was likely to be prescribed as prophylactic treatment).

Statistical analyses were performed using STATA version 12 (STATA Corp, College Station, TX).

\section{Patient and public involvement}

This project was developed within a context of strong patient and public involvement already established within our research team, university, and trust. Two former patients aged 65 and older reviewed the protocol. Their input helped to refine the research question and to improve the protocol considerably. The dissemination plan targets a wide audience, including members of the public, patients, health professionals, and experts in the specialty through various channels available: written communication, events and conferences, networks and social media.

\section{Results}

From the CPRD database we extracted 1577324 observations relating to a primary care UTI consultation between 1 November 2007 and 31 May 2015 for patients aged 65 and older. After applying our exclusion criteria and removing all duplicates, our analytical sample included 312896 distinct UTI episodes diagnosed among 157264 unique patients. An average of two episodes of UTI for each patient were observed in this cohort (fig 2).

The mean age of the study cohort was 76.7 years (SD 9.2 years). At the time of the initial UTI diagnosis, $246630(78.8 \%)$ participants were women, 40.3\% $(n=126215)$ originated from the south of England, and $28.9 \%$ ( $n=90464)$ were from the most deprived areas (index of multiple deprivation fourth and fifth fifths). Overall, $24.2 \%$ of the participants $(n=75563)$ had a Charlson comorbidity index score of 1 or greater, and $22.0 \%(n=68967)$ of the participants had recurrent UTIs (table 1).

For $7.2 \%(n=22534)$ of the UTI episodes a record of an antibiotic prescription in primary care was lacking and 6.2\% $(n=19292)$ were related to a delay in antibiotic prescribing. For those participants prescribed antibiotics for a UTI episode, $73.8 \%$ $(n=200078)$ received either trimethoprim (54.7\%; $\mathrm{n}=148333)$ or nitrofurantoin $(19.1 \% ; \mathrm{n}=51745)$; cephalosporins or amoxicillin/clavulanic acid were prescribed for $11.5 \%(n=31090)$ and $9.5 \%(n=25616)$ of these episodes, respectively, whereas quinolones were prescribed in 4.4\% $(n=11995)$. Pivmecillinam, which was only included among recommended first line treatments in PHE guidelines in October 2014, was prescribed for $0.4 \%(n=1084)$ of these episodes (table 2). ${ }^{27}$ 


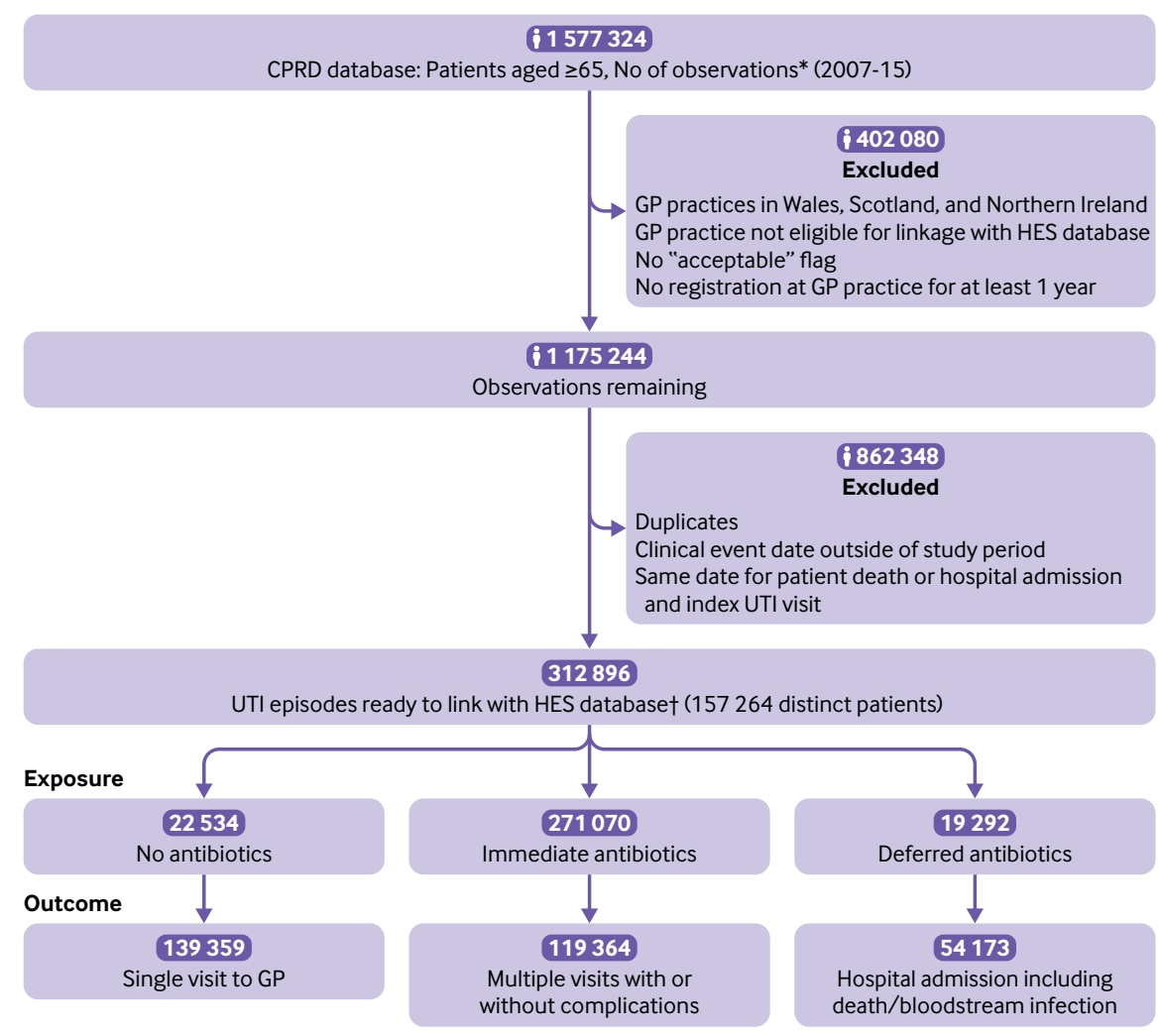

Fig 2 | Flowchart of study cohort. *Observations define all general practitioner (GP) contacts (rows in database) in Clinical Research Datalink database (CPRD). HUTI episode contains all GP contacts that define a single event of UTI for a patient. UTI episode includes 30 day period before diagnosis and 60 day follow-up period after diagnosis. HES=hospital episodes statistics

Patients older than 85 years, living in a deprived area, with a high Charlson comorbidity index score, were mainly managed using either deferred antibiotics or a no antibiotics approach, whereas patients aged between 65 and 74 years were mainly prescribed immediate antibiotics. The female:male ratio was also much higher in the immediate antibiotics group compared with the other groups. A course of antibiotics was more often prescribed at the first visit to the GP or with a delay in patients experiencing recurrent UTIs. Patients who were prescribed antibiotics or were discharged to the hospital within 30 days before the index UTI event were more often prescribed deferred antibiotics or no antibiotics (table 1).

Overall, $7.5 \% \quad(n=23502)$ of the UTI episodes involved at least one of a range of specific or nonspecific signs or symptoms within 30 days before the index UTI. Pain, dysuria, micturition frequency, incontinence, and haematuria were the five most frequent symptoms encountered 30 days before a UTI was diagnosed, and 90.8\% (19 666/21668) of the participants with these symptoms recorded were prescribed antibiotics. This proportion was lower for participants with non-specific signs such as confusion (77.9\%; 136/1459) and malaise (86.6\%; 596/688) (table 1).

Overall, 1539 episodes of bloodstream infection $(0.5 \%$ of total number of UTIs) were recorded in the CPRD or hospital episode statistics, or both within 60 days after a diagnosis of UTI in older people between 2007 and 2015. The rate of bloodstream infection significantly increased when patients were not prescribed antibiotics for their UTI $(2.9 \% v 0.2 \%$ for immediateantibiotics and $2.2 \%$ for deferred antibiotics, $\mathrm{P}<0.001)$. After adjusting for covariates, participants in the deferred antibiotics and no antibiotics groups were significantly more likely to experience a bloodstream infection within 60 days compared with participants in the immediate antibiotics group (adjusted odd ratio 7.12, 95\% confidence interval 6.22 to 8.14 and 8.08 , 7.12 to 9.16 , respectively) (table 3 ).

The number needed to harm ( $\mathrm{NNH}$ ) estimate for bloodstream infection was lower (greater risk) with no antibiotics $(\mathrm{NNH}=37)$ than with deferred antibiotics $(\mathrm{NNH}=51)$, which means that on average for every 37 patients in the no antibiotic group and for every 51 patients in the deferred antibiotic group, one case of bloodstream infection would occur that would not have been seen with use of immediate antibiotics. No significant difference was observed between the rate of bloodstream infection for immediate trimethoprim treatment (233/148333: $0.2 \%)$ and nitrofurantoin treatment (90/51 745: $0.2 \%, \mathrm{P}=0.41$ ).

The proportion of patients admitted to hospital after a UTI episode was nearly two times higher for those in the no antibiotics group (27.0\%) and deferred antibiotics group (26.8\%) compared with those in the immediate antibiotics (14.8\%) group. Among 


\begin{tabular}{|c|c|c|c|c|c|}
\hline Characteristics & $\begin{array}{l}\text { No with UTI } \\
(\mathrm{n}=312896)\end{array}$ & $\begin{array}{l}\text { Immediate antibiotics } \\
(\mathrm{n}=271070)\end{array}$ & $\begin{array}{l}\text { Deferred antibiotics } \\
(n=19292)\end{array}$ & $\begin{array}{l}\text { No antibiotics } \\
(n=22534)\end{array}$ & $P$ value \\
\hline Mean (SD) age (years) & $76.9(9.2)$ & $76.3(9.1)$ & $79.1(9.2)$ & $79.3(9.5)$ & \\
\hline \multicolumn{6}{|l|}{ Age group (years): } \\
\hline $65-74$ & $136175(43.5)$ & $122458(45.2)$ & 6402 (33.2) & $7315(32.5)$ & \multirow[t]{3}{*}{$<0.001$} \\
\hline $75-84$ & $107485(34.3)$ & $92856(34.3)$ & $6881(35.7)$ & $7748(34.4)$ & \\
\hline$\geq 85$ & $69236(22.1)$ & $55756(20.6)$ & $6009(31.1)$ & $7471(33.1)$ & \\
\hline \multicolumn{6}{|l|}{ Sex: } \\
\hline Women & $246630(78.8)$ & $217843(80.4)$ & $13657(70.8)$ & $15130(67.1)$ & \multirow[t]{2}{*}{$<0.001$} \\
\hline Men & $66266(21.2)$ & 53227 (19.6) & $5635(29.2)$ & $7404(32.9)$ & \\
\hline \multicolumn{6}{|l|}{ Region: } \\
\hline North of England and Yorkshire & $65649(21.0)$ & $56744(20.9)$ & $4178(21.7)$ & $4727(21.0)$ & \multirow[t]{4}{*}{$<0.001$} \\
\hline Midlands and east of England & $89337(28.6)$ & $76695(28.3)$ & $5809(30.1)$ & $6833(30.3)$ & \\
\hline South of England & $126215(40.3)$ & $110123(40.6)$ & $7457(38.6)$ & $8635(38.3)$ & \\
\hline London & $31695(10.1)$ & $27508(10.1)$ & $1848(9.6)$ & $2339(10.4)$ & \\
\hline \multicolumn{6}{|l|}{ Index of multiple deprivation (fifths): } \\
\hline 1st (least deprived) & $77945(24.6)$ & $67081(24.8)$ & $4668(24.2)$ & $5196(23.1)$ & \multirow[t]{5}{*}{$<0.001$} \\
\hline 2nd & $75949(24.3)$ & $66084(24.4)$ & $4589(23.8)$ & $5276(23.4)$ & \\
\hline $3 r d$ & $69407(22.2)$ & $60277(22.2)$ & $4193(21.7)$ & 4937 (21.9) & \\
\hline 4th & $51396(16.4)$ & $44239(16.3)$ & $3229(16.7)$ & $3928(17.4)$ & \\
\hline 5th (most deprived) & $39068(12.5)$ & $33279(12.3)$ & $2603(13.5)$ & $3186(14.1)$ & \\
\hline Mean (SD) Charlson comorbidity index score & $0.36(0.8)$ & $0.35(0.7)$ & $0.44(0.9)$ & $0.44(0.9)$ & $<0.001$ \\
\hline Charlson comorbidity index score $\geq 1$ & $75563(24.2)$ & $63694(23.6)$ & $5492(28.5)$ & $6377(27.9)$ & $<0.001$ \\
\hline Immunosuppression & $82(0.03)$ & $67(0.02)$ & $8(0.04)$ & $7(0.03)$ & 0.348 \\
\hline Renal disease & $10215(3.3)$ & $8,588(3.2)$ & $746(3.9)$ & $881(3.9)$ & $<0.001$ \\
\hline Smoking & $12449(4.0)$ & $10798(4.0)$ & $751(3.9)$ & $900(4.0)$ & 0.818 \\
\hline Recurrent UTI & $68967(22.0)$ & $59456(21.9)$ & $6072(31.5)$ & $3439(15.3)$ & $<0.001$ \\
\hline Indwelling urethral catheter & $2627(0.8)$ & $1933(0.7)$ & $352(1.8)$ & $342(1.5)$ & $<0.001$ \\
\hline $\begin{array}{l}\text { Hospital admission within } 30 \text { days before UTI } \\
\text { diagnosis }\end{array}$ & $35825(11.4)$ & $22930(8.5)$ & $5252(27.2)$ & $7643(33.9)$ & $<0.001$ \\
\hline $\begin{array}{l}\text { Antibiotics exposure } 30 \text { days before UTI } \\
\text { diagnosis }\end{array}$ & $61832(19.8)$ & $49079(18.1)$ & $7173(37.2)$ & $5580(24.8)$ & $<0.001$ \\
\hline $\begin{array}{l}\text { Symptoms within } 30 \text { days before UTI } \\
\text { diagnosist: }\end{array}$ & $23502(7.5)$ & $19172(7.1)$ & $2021(10.5)$ & $2309(10.2)$ & \\
\hline Enuresis & $13(0.01)$ & $10(76.9)$ & $3(23.1)$ & 0 & $<0.001$ \\
\hline Offensive urine & $50(0.02)$ & $43(86)$ & $5(10)$ & $2(4)$ & \\
\hline Urgency & $397(0.1)$ & $348(87.7)$ & $23(5.8)$ & $26(6.5)$ & \\
\hline Malaise & $688(0.2)$ & $527(76.6)$ & $69(10.0)$ & $92(13.4)$ & \\
\hline Fatigue & $694(0.2)$ & $607(87.5)$ & $39(5.6)$ & $48(6.9)$ & \\
\hline Confusion & $1459(0.5)$ & $895(61.3)$ & $241(16.5)$ & $323(22.1)$ & \\
\hline Haematuria & $2065(0.7)$ & $1621(78.5)$ & $205(9.9)$ & $239(11.6)$ & \\
\hline Incontinence & $2159(0.7)$ & $1783(82.6)$ & $194(9.0)$ & $182(8.4)$ & \\
\hline Micturition frequency & $3682(1.2)$ & $3151(85.6)$ & $261(7.1)$ & $270(7.3)$ & \\
\hline Dysuria & $4158(1.3)$ & $3411(82.0)$ & $398(9.6)$ & $349(8.4)$ & \\
\hline Pain* & $9604(3.1)$ & $7896(82.2)$ & $746(7.8)$ & $962(10.0)$ & \\
\hline \multicolumn{6}{|l|}{ Outcome: } \\
\hline No (\%) with bloodstream infection (95\% Cl) & $1539(0.5 ; 0.5$ to 0.5$)$ & $479(0.2 ; 0.1$ to 0.2$)$ & $413(2.2 ; 1.9$ to 2.4$)$ & $647(2.9 ; 2.7$ to 3.1$)$ & $<0.001$ \\
\hline No (\%) admitted to hospital $(95 \% \mathrm{Cl})$ & $51261(16.4,16.2$ to 16.5$)$ & $40022(14.8,14.6$ to 14.9$)$ & $5165(26.8,26.2$ to 27.4$)$ & $6074(27.0,26.4$ to 27.5$)$ & $<0.001$ \\
\hline Mean (SD) length of stay (days) & $7.1(15.0)$ & $6.3(14.0)$ & $7.7(13.2)$ & $12.1(20.9)$ & $<0.001$ \\
\hline No (\%) of deaths at 60 days $(95 \% \mathrm{Cl})$ & $6193(2.0,1.9$ to 2.0$)$ & $4431(1.6,1.6$ to 1.7$)$ & $545(2.8,2.6$ to 3.1$)$ & $1217(5.4,5.1$ to 5.7$)$ & $<0.001$ \\
\hline
\end{tabular}

*Pain in locations linked with UTI excluding dysuria.

+Number of UTI episodes where patients reported having at least one symptom within 30 days before the UTI diagnosis. Patients could report one or more symptoms, so the number of episodes for each symptom aggregated across all symptoms differ from the total of participants who experienced one or more symptoms during an episode. In this section, all but the first column are row $\%$, which differ from the rest of the table.

cases admitted to hospital, the length of stay was significantly higher for the no antibiotics group (12.1 days $v 6.3$ days for immediate antibiotics group and 7.7 days for deferred antibiotics group) (table 1 ).

Finally, $2.0 \%(6193 / 312896)$ of the participants older than 65 years who presented to their GP with a UTI died within 60 days; 5.4\% (1217/22 534) for no antibiotics, 2.8\% (545/19292) for deferred antibiotics, and 1.6\% (4431/271070) for immediate antibiotics (table 1). The NNH estimate for death within 60 days was lower with no antibiotics $(\mathrm{NNH}=27)$ than with deferred antibiotics ( $\mathrm{NNH}=83)$, with a calculated risk relative to immediate antibiotics. The Kaplan-Meier curves showed a significant reduction of the 60 day survival for older adults prescribed no antibiotics or deferred antibiotics compared with those prescribed immediate antibiotics (fig 3). Among the patients who were prescribed immediate antibiotics, a small but significant reduction of the 60 day survival was also observed for patients treated with trimethoprim (98.5\%) compared with nitrofurantoin (98.7\%, $\mathrm{P}<0.001$ ) (fig 3). 


\begin{tabular}{|c|c|}
\hline Antibiotics & No $(\%)(n=271070)$ \\
\hline Trimethoprim & $148333(54.7)$ \\
\hline Nitrofurantoin & $51745(19.1)$ \\
\hline Cephalosporins & $31090(11.5)$ \\
\hline Amoxicillin/clavulanic acid & $25616(9.4)$ \\
\hline Quinolones & $11995(4.4)$ \\
\hline Pivmecillinam & $1084(0.4)$ \\
\hline Macrolides & $747(0.3)$ \\
\hline Penicillinase resistant penicillins & $323(0.1)$ \\
\hline Benzylpenicillin and phenoxymethylpenic & $70(0.03)$ \\
\hline Aminoglycosides & $27(0.01)$ \\
\hline Clindamycin & $3(<0.01)$ \\
\hline Carbapenems & $3(<0.01)$ \\
\hline Polymyxin & $1(<0.01)$ \\
\hline
\end{tabular}

The multivariable Cox regression analysis showed that compared with immediate antibiotics and after adjusting for covariates the risk of all cause mortality in older adults at any time during the 60 days of followup was 1.16 times higher with deferred antibiotics (adjusted hazard ratio 1.16, 95\% confidence interval 1.06 to 1.27 ) and 2.18 times higher with no antibiotics (2.18, 2.04 to 2.33) (table 4). The sensitivity analyses excluding the antibiotic treatments with a duration of more than 21 days and 28 days showed consistent results. However, the magnitude of the associations between treatment groups were slightly higher for those prescribed deferred antibiotics $(1.19,1.14$ to 1.23 and $1.36,1.22$ to 1.48 , respectively) and no antibiotics (2.47, 2.28 to 2.63 and $2.61,2.38$ to 2.75 ) compared with those prescribed immediate antibiotics.

In the multivariable Cox regression model, being older, male, living in a deprived area, having a higher Charlson comorbidity index score, being a smoker, being immunosuppressed, having renal disease, and having been exposed to antibiotics and/or discharged

\begin{tabular}{|c|c|c|c|c|}
\hline Variables & $\begin{array}{l}\text { Unadjusted odds ratio } \\
(95 \% \mathrm{Cl})^{\star}\end{array}$ & $P$ value & $\begin{array}{l}\text { Adjustedt odds ratio } \\
(95 \% \mathrm{Cl})^{\star}\end{array}$ & $P$ value \\
\hline \multicolumn{5}{|l|}{ Antibiotic exposure: } \\
\hline Antibiotic at first visit & Reference & & Reference & \\
\hline Deferred antibiotic & $12.36(10.81$ to 14.13$)$ & $<0.001$ & $7.12(6.22$ to 8.14$)$ & $<0.001$ \\
\hline No antibiotic & $16.70(14.81$ to 18.83$)$ & $<0.001$ & $8.08(7.12$ to 9.16$)$ & $<0.001$ \\
\hline \multicolumn{5}{|l|}{ Age group (years): } \\
\hline $65-74$ & Reference & & Reference & \\
\hline $75-84$ & $2.37(2.08$ to 2.71$)$ & $<0.001$ & $1.59(1.39$ to 1.82$)$ & $<0.001$ \\
\hline$\geq 85$ & $3.13(2.73$ to 3.58$)$ & $<0.001$ & 1.67 (1.44 to 1.93$)$ & $<0.001$ \\
\hline \multicolumn{5}{|l|}{ Sex: } \\
\hline Men & Reference & & Reference & \\
\hline Women & $0.25(0.23$ to 0.28$)$ & $<0.001$ & $0.45(0.40$ to 0.50$)$ & $<0.001$ \\
\hline \multicolumn{5}{|l|}{ Region: } \\
\hline North of England and Yorkshire & Reference & & & \\
\hline Midlands and East of England & $1.02(0.89$ to 1.18$)$ & 0.74 & & \\
\hline South of England & $0.86(0.75$ to 0.99$)$ & 0.03 & & \\
\hline London & $0.90(0.74$ to 1.09$)$ & 0.28 & & \\
\hline \multicolumn{5}{|l|}{ Index of multiple deprivation (fifths): } \\
\hline 1st (least deprived) & Reference & & Reference & \\
\hline 2 & $1.00(0.86$ to 1.16$)$ & 0.98 & $0.97(0.83$ to 1.14$)$ & 0.74 \\
\hline 3 & $1.07(0.92$ to 1.25$)$ & 0.38 & $1.04(0.89$ to 1.22$)$ & 0.58 \\
\hline 4 & 1.35 (1.15 to 1.58$)$ & $<0.001$ & $1.21(1.03$ to 1.42$)$ & 0.02 \\
\hline 5th (most deprived) & $1.39(1.18$ to 1.65$)$ & $<0.001$ & $1.18(0.99$ to 1.40$)$ & 0.06 \\
\hline Charlson comorbidity index score (0-12) & $1.35(1.29$ to 1.40$)$ & $<0.001$ & $1.10(1.04$ to 1.16$)$ & $<0.001$ \\
\hline Immunosuppressed & $5.06(1.26$ to 20.31$)$ & 0.02 & & \\
\hline Renal disease & 1.61 (1.28 to 2.02$)$ & $<0.001$ & & \\
\hline Smoking & $1.20(0.95$ to 1.52$)$ & 0.16 & & \\
\hline \multicolumn{5}{|l|}{ Year of UTI: } \\
\hline 2007-08 & Reference & & Reference & \\
\hline $2008-09$ & $0.70(0.45$ to 1.10$)$ & 0.12 & $0.67(0.42$ to 1.07$)$ & 0.09 \\
\hline $2009-10$ & $0.74(0.48$ to 1.16$)$ & 0.20 & $0.66(0.42$ to 1.05$)$ & 0.08 \\
\hline 2010-11 & $0.97(0.63$ to 1.51$)$ & 0.90 & $0.86(0.55$ to 1.36$)$ & 0.53 \\
\hline 2011-12 & $0.90(0.58$ to 1.40$)$ & 0.65 & $0.77(0.49$ to 1.22$)$ & 0.26 \\
\hline 2012-13 & $1.98(1.30$ to 3.01$)$ & 0.001 & $1.57(1.01$ to 2.42$)$ & 0.04 \\
\hline 2013-14 & $3.38(2.24$ to 5.12$)$ & $<0.001$ & $2.72(1.77$ to 4.19$)$ & $<0.001$ \\
\hline $2014-15$ & $4.52(2.98$ to 6.83$)$ & $<0.001$ & $3.46(2.25$ to 5.32$)$ & $<0.001$ \\
\hline Symptoms $<30$ days before UTI diagnosis & $1.20(1.01$ to 1.44$)$ & 0.04 & & \\
\hline Antibiotic prescribed $<30$ days before UTI diagnosis & $1.26(1.12$ to 1.42$)$ & $<0.001$ & & \\
\hline Admitted to hospital 30 days before diagnosis & $10.45(9.44$ to 11.57$)$ & $<0.001$ & $3.94(3.54$ to 4.39$)$ & $<0.001$ \\
\hline Indwelling urethral catheter & $3.60(2.66$ to 4.89$)$ & $<0.001$ & & \\
\hline Recurrent UTIs & $0.77(0.67$ to 00.88$)$ & $<0.001$ & $0.86(0.75$ to 0.99$)$ & 0.04 \\
\hline Interaction antibiotic exposure and recurrence & $1.27(1.15$ to 1.41$)$ & $<0.001$ & & \\
\hline
\end{tabular}




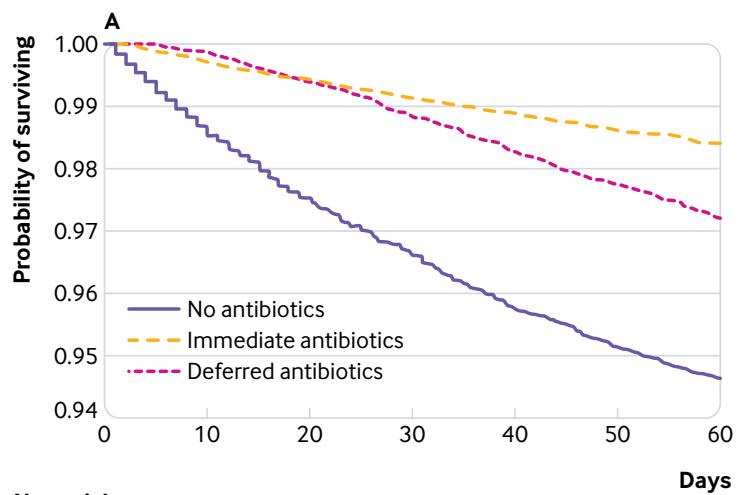

No at risk

No antibiotics

$\begin{array}{lllllll}22534 & 22236 & 21978 & 21783 & 21582 & 21446 & 21327\end{array}$

Immediate antibiotics

$\begin{array}{lllllll}271070 & 270350 & 269498 & 268769 & 268013 & 267360 & 266709\end{array}$

Deferred antibiotics

$\begin{array}{lllllll}19292 & 19262 & 19176 & 19075 & 18963 & 18860 & 18754\end{array}$
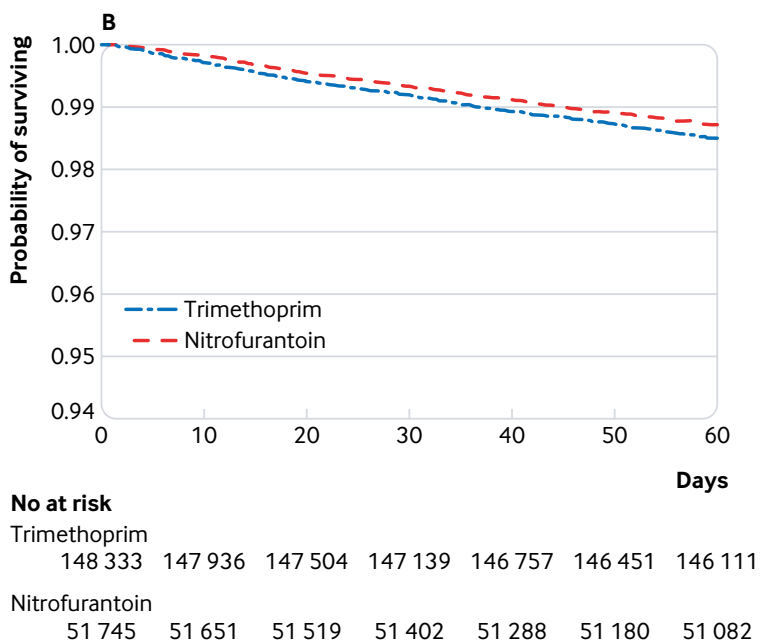

Fig 3 | Kaplan-Meier survival curves by antibiotic management over 60 days

from hospital 30 days before the UTI diagnosis, were all positively associated with 60 day all cause mortality. In contrast, living in London or the south of England compared with the north of England and Yorkshire or the east of England and the Midlands, as well as having recurrent UTIs, were significantly associated with a decrease in mortality. The interaction factor between antibiotic use and recurrent UTIs was also significant and thus included in the final multivariable model (table 4).

Finally, for the care pathway of older adults with a diagnosed UTI in primary care, $44.5 \%$ of patients with a UTI $(n=139359)$ presented only once to the GP without a subsequent hospital admission, whereas $38.2 \%(n=119364)$ required multiple visits to the GP for the same UTI episode and $17.3 \%(n=54173)$ were admitted to hospital within 60 days of their first visit for a UTI. Among patients who were not prescribed antibiotics, 29.5\% $(n=6637)$ were admitted to the hospital or died within 60 days, compared with $16.4 \%$ ( $\mathrm{n}=47536)$ among those who were prescribed antibiotics; $27.3 \%(n=18065)$ of men were admitted to hospital or died within 60 days compared with $14.6 \%$ $(n=36108)$ of women. Older patients with UTI were more likely to be admitted to hospital compared with 65-74 year olds who were more likely to have a single visit to the GP (table 5).

\section{Discussion}

This study has shown that patients aged older than 65 years with a diagnosis of urinary tract infection (UTI) in the community are at significantly increased risk of bloodstream infection and death within 60 days when antibiotic treatment was either not prescribed or deferred.

The odds of developing a bloodstream infection within 60 days was sevenfold and eightfold higher in the deferred antibiotic and no antibiotics groups, respectively, compared with the immediate antibiotics group. The number needed to harm (NNH) for bloodstream infection was lower with no antibiotics $(\mathrm{NNH}=37)$ than with deferred antibiotics $(\mathrm{NNH}=51)$, when both were compared with immediate antibiotics. Patients in the no antibiotics group were also more than twice as likely to die, whereas patients in the deferred antibiotics group were 1.16 as likely to die during the 60 days after a UTI compared with those in the immediate antibiotic group. The NNH estimate for death was lower with no antibiotics $(\mathrm{NNH}=27)$ than with deferred antibiotics $(\mathrm{NNH}=83)$.

These findings were adjusted for potential confounding factors and changes over time to account for updates to national guidelines. The risk of bloodstream infection and all cause mortality also increased for male and older patients, especially those older than 85 years and those living in more deprived areas. Among patients who were prescribed immediate antibiotics for an episode of UTI, a small but significant increase of the 60 day survival was observed for those treated with nitrofurantoin compared with trimethoprim. This increase could reflect either higher levels of resistance to trimethoprim ${ }^{16}$ or a healthier population treated with nitrofurantoin; the latest being not recommended for patients with poor kidney function. ${ }^{28}$ These results are consistent with a recent cohort study using data from Clinical Practice Research Datalink (CPRD), where nitrofurantoin was associated with the smallest odds of death within 14 days of antibiotic initiation for UTI of all the antibiotics investigated. $^{29}$

\section{Strengths and limitations of this study}

A major strength of this study is the use of individual patient level data for adults older than 65 years extracted from a large nationwide general practice records database and linked to hospital and mortality records. This provided the opportunity to track the care pathways of a vulnerable population with a diagnosis of UTI in the community with a 60 day follow-up. The linkage with mortality data from the Office for National Statistics minimised possible bias in the risk estimates of all cause mortality among older adults treated in a routine care setting. 


\begin{tabular}{|c|c|c|c|c|}
\hline Variables & $\begin{array}{l}\text { Unadjusted hazard ratio } \\
(95 \% \mathrm{Cl})^{\star}\end{array}$ & $P$ value & $\begin{array}{l}\text { Adjustedt hazard ratio } \\
(95 \% \mathrm{Cl})^{*}\end{array}$ & $P$ value \\
\hline \multicolumn{5}{|l|}{ Antibiotic exposure: } \\
\hline Antibiotic first visit & Reference & & Reference & \\
\hline Deferred antibiotic & $1.73(1.59$ to 1.89$)$ & $<0.001$ & $1.16(1.06$ to 1.27$)$ & 0.001 \\
\hline No antibiotics & $3.38(3.17$ to 3.60$)$ & $<0.001$ & $2.18(2.04$ to 2.33$)$ & $<0.001$ \\
\hline \multicolumn{5}{|l|}{ Age group (years): } \\
\hline $65-74$ & Reference & & Reference & \\
\hline $75-84$ & $3.20(2.94$ to 3.48$)$ & $<0.001$ & 2.79 (2.60 to 2.99$)$ & $<0.001$ \\
\hline$\geq 85$ & $9.42(8.71$ to 10.19$)$ & $<0.001$ & $7.87(7.37$ to 8.40$)$ & $<0.001$ \\
\hline \multicolumn{5}{|c|}{ 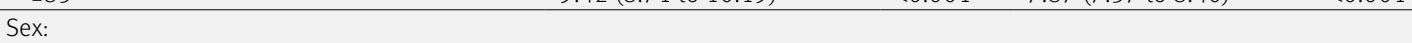 } \\
\hline Men & Reference & & Reference & \\
\hline Women & $0.42(0.40$ to 0.44$)$ & $<0.001$ & $0.52(0.49$ to 0.55$)$ & $<0.001$ \\
\hline \multicolumn{5}{|l|}{ Region: } \\
\hline North of England and Yorkshire & Reference & & Reference & \\
\hline Midlands and east of England & $0.98(0.92$ to 1.06$)$ & 0.67 & $0.97(0.90$ to 1.04$)$ & 0.39 \\
\hline South of England & $0.93(0.87$ to 0.99$)$ & 0.025 & $0.93(0.87$ to 1.00$)$ & 0.05 \\
\hline London & $0.69(0.62$ to 0.77$)$ & $<0.001$ & $0.72(0.65$ to 0.80$)$ & $<0.001$ \\
\hline \multicolumn{5}{|l|}{ Index of multiple deprivation (fifths): } \\
\hline 1st (least deprived) & Reference & & Reference & \\
\hline 2nd & $1.17(1.08$ to 1.26$)$ & $<0.001$ & $1.16(1.07$ to 1.26$)$ & $<0.001$ \\
\hline $3 r d$ & $1.31(1.22$ to 1.42$)$ & $<0.001$ & $1.27(1.18$ to 1.37$)$ & $<0.001$ \\
\hline 4th & $1.29(1.19$ to 1.40$)$ & $<0.001$ & $1.29(1.19$ to 1.40$)$ & $<0.001$ \\
\hline 5th (most deprived) & $1.35(1.23$ to 1.47$)$ & $<0.001$ & $1.33(1.21$ to 1.45$)$ & $<0.001$ \\
\hline Charlson comorbidity index score (0-12) & $1.50(1.47$ to 1.53$)$ & $<0.001$ & $1.27(1.21$ to 1.33$)$ & $<0.001$ \\
\hline Immunosuppressed & $5.26(2.60$ to 10.65$)$ & $<0.001$ & $5.09(2.54$ to 10.20$)$ & $<0.001$ \\
\hline Renal disease & $2.00(1.81$ to 2.22$)$ & $<0.001$ & $1.81(1.72$ to 1.93$)$ & 0.002 \\
\hline Smoking & $0.82(0.71$ to 0.94$)$ & 0.005 & $1.27(1.10$ to 1.46$)$ & 0.001 \\
\hline \multicolumn{5}{|l|}{ Year of UTI: } \\
\hline 2007-08 & Reference & & Reference & \\
\hline 2008-09 & $1.20(1.00$ to 1.45$)$ & 0.05 & $1.14(0.94$ to 1.37$)$ & 0.18 \\
\hline $2009-10$ & $1.17(0.97$ to 1.41$)$ & 0.09 & $1.09(0.89$ to 1.30$)$ & 0.42 \\
\hline 2010-11 & $1.25(1.04$ to 1.51$)$ & 0.02 & $1.13(0.92$ to 1.36$)$ & 0.27 \\
\hline 2011-12 & $1.29(1.07$ to 1.55$)$ & 0.008 & 1.09 (0.91 to 1.32$)$ & 0.36 \\
\hline $2012-13$ & $1.50(1.25$ to 1.81$)$ & $<0.001$ & $1.23(1.02$ to 1.48$)$ & 0.02 \\
\hline 2013-14 & $1.55(1.29$ to 1.87$)$ & $<0.001$ & $1.24(1.03$ to 1.50$)$ & 0.02 \\
\hline $2014-15$ & $1.58(1.30$ to 1.91$)$ & $<0.001$ & $1.23(1.02$ to 1.48$)$ & 0.04 \\
\hline Symptoms $<30$ days before UTI diagnosis & $1.23(1.13$ to 1.34$)$ & $<0.001$ & & \\
\hline Antibiotic prescribed $<30$ days before UTI diagnosis & $1.50(1.42$ to 1.58$)$ & $<0.001$ & $1.31(1.22$ to 1.42$)$ & $<0.001$ \\
\hline Admitted to hospital 30 days before diagnosis & 3.24 (3.07 to 3.42$)$ & $<0.001$ & $1.88(1.77$ to 2.03$)$ & $<0.001$ \\
\hline Indwelling urethral catheter & $2.71(2.29$ to 3.21$)$ & $<0.001$ & & \\
\hline Recurrent UTIs & $0.92(0.87$ to 0.98$)$ & 0.008 & $0.89(0.82$ to 0.95$)$ & $<0.001$ \\
\hline Interaction antibiotic exposure and recurrence & $1.11(1.06$ to 1.17$)$ & $<0.001$ & 1.19 (1.10 to 1.30$)$ & $<0.001$ \\
\hline
\end{tabular}

The large sample size of about 160000 patients with more than 300000 distinct UTI episodes substantially increased the power of the analyses, especially for rare severe adverse events in older adults (ie, bloodstream infection, mortality). As the base population is representative of the English general population, our results are generalisable to the entire English population of elderly patients.

In addition, records were routinely collected by GPs in normal care settings providing an unbiased selection of both the exposed and the control cohorts and reducing the opportunity for information bias (as exposure and outcomes were prospectively collected independently). This study not only helped us to understand the management of UTI in an older population in real life but also enabled us to assess the no antibiotic treatment approach for UTI. This option would have been challenging in a prospective trial because of ethical restraints. Finally, we had access to detailed patient information, including patient diagnoses, comorbidities, prescribed drugs, and procedures, allowing us to control for the effects of several potential confounders in the multivariable regression models.

The main limitations of our study are common to observational studies using routinely collected electronic health record data, and include unmeasured and residual confounders, missing data and potential biases, such as confounding by indication, misclassification biases, or inconsistencies in coding within and between practices and over time.

Patients were identified and included in our study based on a clinical diagnosis recorded using a coding system. Therefore, most of the cases were suspected UTIs, with only a minority based on a laboratory confirmed diagnosis. Separate microbiology data 


\begin{tabular}{|c|c|c|c|c|c|}
\hline Variables & No of participants & Single visit to GP & Multiple visits to GP & $\begin{array}{l}\text { Hospital admission including } \\
\text { death within } 60 \text { days }\end{array}$ & $P$ value \\
\hline \multicolumn{6}{|c|}{ Antibiotic exposure*: } \\
\hline No antibiotics & $22534(7.2 ; 7.1$ to 7.3$)$ & $14722(65.33 ; 64.71$ to 65.95$)$ & $1175(5.2 ; 4.9$ to 5.5$)$ & $6637(29.5 ; 28.9$ to 30.1$)$ & \multirow[t]{2}{*}{$<0.001$} \\
\hline Antibiotics* & $290362(92.8 ; 92.7$ to 92.9$)$ & $124637(42.9 ; 42.7$ to 43.1$)$ & $118189(40.7 ; 40.5$ to 40.9$)$ & $47536(16.4 ; 16.2$ to 16.5$)$ & \\
\hline \multicolumn{6}{|l|}{ Sex: } \\
\hline Men & $66266(21.2 ; 21.0$ to 21.3$)$ & $24561(37.1 ; 36.7$ to 37.4$)$ & $23640(35.7 ; 35.3$ to 36.0$)$ & $18065(27.3 ; 26.9$ to 27.6$)$ & \multirow[t]{2}{*}{$<0.001$} \\
\hline Women & $246630(78.8 ; 78.7$ to 79.0$)$ & $114798(46.6 ; 46.4$ to 46.7$)$ & $95724(38.8 ; 38.6$ to 39.0$)$ & $36108(14.6 ; 14.5$ to 14.8$)$ & \\
\hline \multicolumn{6}{|l|}{ Age group (years): } \\
\hline $65-74$ & $136175(43.5 ; 43.4$ to 43.7$)$ & $66972(49.2 ; 48.9$ to 49.5$)$ & $51336(37.7 ; 37.4$ to 38.0$)$ & $17867(13.1 ; 12.9$ to 13.3$)$ & \multirow[t]{3}{*}{$<0.001$} \\
\hline $75-84$ & $107485(34.4 ; 34.2$ to 34.5$)$ & $45909(42.7 ; 42.4$ to 43.0$)$ & $41427(38.5 ; 38.3$ to 38.83 & $20149(18.8 ; 18.5$ to 19.0$)$ & \\
\hline$\geq 85$ & $69236(22.1 ; 22.0$ to 22.3$)$ & $26478(38.2 ; 37.9$ to 38.6$)$ & $26601(38.4 ; 38.1$ to 38.8$)$ & $16157(23.3 ; 23.02$ to 23.7$)$ & \\
\hline Total & 312896 & $139359(44.5 ; 44.4$ to 44.7$)$ & $119364(38.2 ; 38.0$ to 38.3$)$ & $54173(17.3 ; 17.2$ to 17.5$)$ & \\
\hline
\end{tabular}

with UTI confirmation and drug sensitivities were unavailable. We used a pragmatic approach to include all the possible descriptors a GP might use for infectious disease of the urinary tract. Further research using a more specific list of codes for UTI could be worth exploring.

The uncertainties around the UTI diagnosis in elderly patients as a result of uncommon presentations might have biased the selection of our initial cohort of patients with UTI. A variety of acute infectious or noninfectious causes leading to those uncertainties might have driven the adverse outcomes. CPRD only reports the symptoms documented by GPs and does not always include a structured assessment of the illness with information on symptom severity and onset, for example, which made the control for confounding by indication difficult. However, we may have observed that the protective effect of immediate antibiotics would exceed the effect of confounding by indication as described by Little et al. ${ }^{30}$

There were potential classification biases for the exposure variable associated with the lack of information on treatment compliance by the patients and on delayed prescriptions issued by GPs at the index visit in CPRD. The database did not define whether the antibiotics prescribed on the date of the initial UTI diagnosis had to be taken immediately or several days later in the context of ongoing symptoms. This common delayed prescription strategy to reduce inappropriate antibiotic prescribing, as well as patients who did not consume the antibiotics prescribed by the GPs, may have incorrectly classified some patients as belonging to the immediate antibiotics group. Conversely, the database did not identify patients who had already accessed antibiotics (rescue pack or previous prescription). This might partly explain the observation (see table 1) that more patients in the deferred and no antibiotics groups had received antibiotics or had been admitted to the hospital in the previous month compared with the immediate antibiotics group. We also did not consider the number of days between the date of the initial UTI diagnosis and the date of deferred antibiotics when a prescription was not issued at the index visit, which may have an impact on the adverse outcomes.
We cannot exclude an alternative non-urinary source for the bloodstream infections. The origin of the bloodstream infections is not often specified in hospital episode statistics or CPRD. In the context of the cohort of patients in this study initially having a diagnosis of a UTI in primary care, most of the bloodstream infections recorded should have a urinary source. Reverse causality was unlikely in this study as we have tried to make sure that the date of the exposure (antibiotic management) was before the outcomes (bloodstream infections or mortality, or both).

Finally, the complexity of the coding system in electronic health record databases, the variability in recording information, as well as missing data, might have also prevented us from capturing a comprehensive list of the complications related to UTI and the confounders associated with increased risk of bloodstream infection and all cause mortality. For example, some nursing homes may have a wait and see policy for antibiotic prescribing to prevent Clostridium difficile outbreaks. It is also possible that patients with cognitive impairment lack insight into the severity of their illness and were not prescribed antibiotics while they were needed. By adjusting our outcomes on existing comorbidities using the Charlson comorbidity index, we have tried to minimise the presence of some residual confounders.

\section{Comparison with existing literature}

This study comprised a large sample size population, assessing the real life care management, including no antibiotics and deferred antibiotics, as well as the outcomes and care pathway of older adults with a diagnosis of UTI in primary care. Limited evidence is available to support the choice of no antibiotics or of deferred antibiotics for the management of UTI in primary care, as ethical concerns have prevented placebo controlled studies for UTI. ${ }^{31}$

A systematic review of randomised controlled trials showed that antibiotic treatment is more effective at achieving faster symptom relief, microbiological clearance, and lower reinfection rates than placebo for uncomplicated cystitis in women aged 15 to 84 years. ${ }^{32}$ However, potential unintended adverse events have not been explored (eg, admission to hospital, 
bloodstream infection, or death) as a large sample size would be needed to capture these rare serious adverse events. Another randomised controlled trial, which evaluated the efficacy of initial symptomatic treatment with ibuprofen versus immediate antibiotic treatment in uncomplicated UTI for women younger than 65 years has shown an increase in the total burden of symptoms and pyelonephritis cases in the ibuprofen arm. $^{33}$

In the context of randomised controlled trials, strict exclusion criteria particularly related to age have been applied that prevent the results being generalised to older adult populations who may require a different approach in the management of UTI. In contrast, our study specifically looked at the group of older patients ( $>65$ years) who are more susceptible to complications and are often neglected in UTI related research. We showed that antibiotics prescribed at the time of UTI diagnosis may benefit this vulnerable population by significantly reducing the risk of all cause mortality and the rate of bloodstream infection and hospital admission.

Recent guidance from the National Institute of Health and Care Excellence has proposed no antibiotic or delayed antibiotic prescriptions when infection is likely to be self limiting in an effort to reduce inappropriate prescribing. ${ }^{15} 34$ Evidence and recommendations, however, refer mainly to upper respiratory tract infections. ${ }^{3035}$ Evidence is nonetheless emerging that delayed prescribing in the treatment of UTI is becoming more acceptable in practice. ${ }^{3637}$

A randomised controlled trial evaluated various antibiotic management strategies, including empirical delayed (by 48 hours) antibiotics and immediate antibiotics strategies for UTI in women younger than 70 years. No significant differences in symptom duration, severity, or frequency of symptoms between the strategies were reported. ${ }^{38}$ In our study, deferred antibiotics were associated with less severe adverse outcomes than no antibiotics for older adults but still showed a significantly higher risk of mortality compared with immediate antibiotics.

The question remains as to why a significant proportion (about 7\%) of vulnerable older patients had a diagnosis of UTI but were not prescribed antibiotics. It could be patient or doctor choice, but it is also possible that antimicrobial stewardship programmes and quality premium payments are encouraging a culture of more judicious antibiotic use. Public Health England recently reported a $13.2 \%$ reduction in antibiotic prescribing in primary care between 2013 and $2017 .^{16}$

There is also a major concern about the risk of $C$ difficile infection in elderly people associated with antibiotic use, which also includes trimethoprim. ${ }^{39} 40$

Other circumstances, such as the presence of mild urinary symptoms, may encourage clinicians to withhold antibiotics in the context of a working diagnosis of UTI. Nevertheless, if this explanation holds true, patients with disease not severe enough to prompt antibiotic treatment are at risk of severe consequences.

\section{Clinical, policy, and research implications}

Our findings suggest that GPs consider early prescription of antibiotics for this vulnerable group of older adults in view of their increased susceptibility to sepsis after UTI and despite a growing pressure to reduce inappropriate antibiotic use. Particular care is needed for the management of older men and those in deprived communities. For researchers, there is a need to improve the understanding of the effects of deferred antibiotic prescribing in routine practice. New medical record or retrievable codes should therefore be in place to record when primary care clinicians advise patients to delay antibiotic consumption.

\section{Conclusion}

Results from this large population based cohort study suggest a significant increase in the risk of bloodstream infection and all cause mortality and the rate of hospital admission associated with no antibiotics and deferred antibiotics compared with immediate antibiotics in older adults with a diagnosis of UTI in primary care. Our study suggests the early initiation of antibiotics for UTI in older high risk adult populations (especially men aged $>85$ years) should be recommended to prevent serious complications.

We thank Anthony Thomas, data manager for the Dr Foster Unit at Imperial College London and Masha Mazidi, database manager at the Primary Care and Public Health Department Imperial College London, for helping and facilitating with the use of CPRD. This work uses data provided by patients and collected by the National Health Service as part of their care and support.

Contributors: $M G$ is first author. MG, HL, RG, PA, MM, and AP designed the study. MG, HL, and RG wrote the protocol. MG, JHD, $\mathrm{HL}, \mathrm{RG}$, and MM carried out the data management and the code list identification. MG and JHD carried out the statistical analysis and wrote the first draft. All the authors contributed substantially to drafts and critical revision and approved the final manuscript. PA is the guarantor. The corresponding author attests that all listed authors meet authorship criteria and that no others meeting the criteria have been omitted.

Funding: This work was supported by the National Institute for Health Research Health Protection Research Unit (NIHR HPRU) (grant No HPRU-2012-10 047) in Healthcare Associated Infections and Antimicrobial Resistance at Imperial College London in partnership with Public Health England (PHE), and the National Institute for Health Research (NIHR) Biomedical Research Centre at Guy's and St Thomas' NHS Foundation Trust and King's College London. The NIHR HPRU is also affiliated with the National Institute of Health Research (NIHR) Imperial Patient Safety Translational Research Centre. The Department of Primary Care and Public Health at Imperial College London is grateful for support from the NW London NIHR Collaboration fo Leadership in Applied Health Research and Care (CLAHRC) and the Imperial NIHR Biomedical Research Centre. The views expressed are those of the author(s) and not necessarily those of the NHS, NIHR, Department of Health, or Public Health England.

Competing interests: All authors have completed the ICMJE uniform disclosure form at http://www.icmje.org/coi disclosure.pdf and declare: grants support from NIHR and Dr Foster Intelligence for the submitted work; no financial relationships with any organisations that might have an interest in the submitted work in the previous three years; no other relationships or activities that could appear to have influenced the submitted work except for MG who declares working as an epidemiologist at GSK in therapeutic areas not related to the submitted work. 
Ethical approval: The protocol for research using the Clinical Practice Research Datalink (CPRD) was approved by the Independent Scientific Advisory Committee (CPRD No 16_026R).

Data sharing: CPRD data were provided under a licence that does not permit sharing. Data are, however, obtainable from CPRD under standard conditions.

Transparency: The manuscript's guarantor (PA) affirms that the manuscript is an honest, accurate, and transparent account of the study being reported; that no important aspects of the study have been omitted; and that any discrepancies from the study as planned (and, if relevant, registered) have been explained.

This is an Open Access article distributed in accordance with the terms of the Creative Commons Attribution (CC BY 4.0) license, which permits others to distribute, remix, adapt and build upon this work, for commercial use, provided the original work is properly cited. See: http://creativecommons.org/licenses/by/4.0/.

1 Linhares I, Raposo T, Rodrigues A, Almeida A. Frequency and antimicrobial resistance patterns of bacteria implicated in community urinary tract infections: a ten-year surveillance study (2000-2009). BMC Infect Dis 2013;13:19. doi:10.1186/1471-2334-13-19.

2 Wagenlehner FM, Lichtenstern C, Rolfes C, et al. Diagnosis and management for urosepsis. Int I Urol 2013:20:963-70.

3 Martin GS, Mannino DM, Moss M. The effect of age on the development and outcome of adult sepsis. Crit Care Med 2006:34:15-21. doi:10.1097/01.CCM.0000194535.82812.BA

4 Tal S, Guller V, Levi S, et al. Profile and prognosis of febrile elderly patients with bacteremic urinary tract infection. J Infect 2005:50:296-305. doi:10.1016/j.jinf.2004.04.004

5 Cove-Smith A, Almond MK. Management of urinary tract infections in the elderly. Trends in Urology, Gynaecol Sex Health 2007;12:31-4.

6 Lutters M, Vogt-Ferrier NB. Antibiotic duration for treating uncomplicated, symptomatic lower urinary tract infections in elderly women. Cochrane Database Syst Rev 2008;(3):CD001535. doi:10.1002/14651858.CD001535.pub2.

7 Lim VH, Whitehurst T, Usoro E, Ming Ng S. Management of urinary tract infections in elderly patients: Strategies for improvement. BMJ Qual Improv Rep 2014;3:u203314.w1503. doi:10.1136/bmjquality. u203314.w1503.

8 McMurdo ME, Gillespie ND. Urinary tract infection in old age: over-diagnosed and over-treated. Age Ageing 2000;29:297-8. doi:10.1093/ageing/29.4.297

9 Crnich CJ, Jump RL, Nace DA. Improving Management of Urinary Tract Infections in Older Adults: A Paradigm Shift or Therapeutic Nihilism? J Am Geriatr Soc 2017;65:1661-3. doi:10.1111/jgs.14961.

10 Nace DA, Drinka PJ, Crnich Cl. Clinical uncertainties in the approach to long term care residents with possible urinary tract infection. J Am Med Dir Assoc 2014;15:133-9. doi:10.1016/j.jamda.2013.11.009.

11 PHE. English surveillance programme for antimicrobial utilisation and resistance (ESPAUR) Report 2016. Public Health England, 2016: 181.

12 Abbo LM, Hooton TM. Antimicrobial Stewardship and Urinary Tract Infections. Antibiotics (Basel) 2014;3:174-92. doi:10.3390/ antibiotics3020174.

13 NICE. CKS Clinical Knowledge Summaries - Urinary tract infection (lower) - women London: National Institute for Health and Care Excellence; 2015 https://cks.nice.org.uk/urinary-tract-infectionlower-women\#!scenario accessed 28/06/2018 18

14 SIGN. Management of suspected bacterial urinary tract infection in adults: a national clinical guideline. Scottish Intercollegiate Guidelines Network. London: Scottish Intercollegiate Guidelines Network; 2012 [updated July 2012. www.sign.ac.uk/assets/sign88. pdf accessed 28/06/2018 2018

15 NICE. Guidelines: Urinary tract infection (lower): antimicrobial prescribing: National Institute for Health and Care Excellence:34.

16 PHE. English surveillance programme for antimicrobial utilisation and resistance (ESPAUR) Report 2018. Public Health England, 2018: 147.

17 NHS_England. Quality Premium: 2016/17 Guidance for Clinical Commissioning Groups (CCGs) London, UK: NHS England; 2016 www.england.nhs.uk/wp-content/uploads/2016/03/qualty-premguid-2016-17.pdf accessed 28/06/2018 2018.

18 Bou-Antoun S, Costelloe C, Honeyford K, et al. Age-related decline in antibiotic prescribing for uncomplicated respiratory tract infections in primary care in England following the introduction of a national financial incentive (the Quality Premium) for health commissioners to reduce use of antibiotics in the community: an interrupted time series analysis. / Antimicrob Chemother 2018;73:2883-92. doi:10.1093/ $\mathrm{jac} / \mathrm{dky} 237$.
19 Balinskaite V, Johnson AP, Holmes A, Aylin P. The impact of a national antimicrobial stewardship programme on antibiotic prescribing in primary care: an interrupted time series analysis. Clin Infect Dis 2018; published online first 18 Oct. doi:10.1093/cid/ciy902.

20 Ahmed H, Farewell D, Jones HM, Francis NA, Paranjothy S, Butler CC. Incidence and antibiotic prescribing for clinically diagnosed urinary tract infection in older adults in UK primary care, 2004-2014. PLoS One 2018;13:e0190521. doi:10.1371/journal.pone.0190521.

21 Herrett E, Gallagher AM, Bhaskaran K, et al. Data Resource Profile: Clinical Practice Research Datalink (CPRD). Int J Epidemiol 2015;44:827-36. doi:10.1093/ije/dyv098.

22 Chisholm J. The Read clinical classification. BMJ 1990;300:1092. doi:10.1136/bmi.300.6732.1092

23 Williams T, van Staa T, Puri S, Eaton S. Recent advances in the utility and use of the General Practice Research Database as an example of a UK Primary Care Data resource. Ther Adv Drug Saf 2012;3:89-99. doi:10.1177/2042098611435911.

24 Duane S, Beatty P, Murphy AW, Vellinga A. Exploring Experiences of Delayed Prescribing and Symptomatic Treatment for Urinary Tract Infections among General Practitioners and Patients in Ambulatory Care: A Qualitative Study. Antibiotics (Basel) 2016;5:E27. doi:10.3390/antibiotics5030027.

25 Charlson ME, Pompei P, Ales KL, MacKenzie CR. A new method of classifying prognostic comorbidity in longitudinal studies: development and validation. / Chronic Dis 1987;40:373-83. doi:10.1016/0021-9681(87)90171-8

26 Mody L, Juthani-Mehta M. Urinary tract infections in older women: a clinical review. JAMA 2014;311:844-54. doi:10.1001/ jama.2014.303.

27 PHE. Management of infection guidance for primary care for consultation and local adaptation. Public Health England, 2014: 61.

28 PHE. Management and treatment of common infections Antibiotic guidance for primary care: For consultation and local adaptation. Public Health England, 2017: 150.

29 Crellin E, Mansfield KE, Leyrat C, et al. Trimethoprim use for urinary tract infection and risk of adverse outcomes in older patients: cohort study. BMJ 2018;360:k341. doi:10.1136/bmj.k341.

30 Little P, Stuart B, Hobbs FD, et al, DESCARTE investigators. Antibiotic prescription strategies for acute sore throat: a prospective observational cohort study. Lancet Infect Dis 2014;14:213-9. doi:10.1016/S1473-3099(13)70294-9

31 World Medical A, World Medical Association. World Medical Association Declaration of Helsinki: ethical principles for medical research involving human subjects. J Postgrad Med 2002;48:206-8.

32 Falagas ME, Kotsantis IK, Vouloumanou EK, Rafailidis PI. Antibiotics versus placebo in the treatment of women with uncomplicated cystitis: a meta-analysis of randomized controlled trials. J Infect 2009;58:91-102. doi:10.1016/j.jinf.2008.12.009.

33 Gágyor I, Bleidorn J, Kochen MM, Schmiemann G, Wegscheider K, Hummers-Pradier E. Ibuprofen versus fosfomycin for uncomplicated urinary tract infection in women: randomised controlled trial. BMJ 2015;351:h6544. doi:10.1136/bmj.h6544.

34 Morley GL, Wacogne ID. UK recommendations for combating antimicrobial resistance: a review of 'antimicrobial stewardship: systems and processes for effective antimicrobial medicine use, (NICE guideline NG15, 2015) and related guidance. Arch Dis Child Educ Pract Ed 2017; edpract-2016-311557.

35 Spurling GK, Del Mar CB, Dooley L, Foxlee R, Farley R. Delayed antibiotics for respiratory infections. Cochrane Database Syst Rev 2013:(4):CD004417.

36 Leydon GM, Turner S, Smith H, Little P, UTIS team. Women's views about management and cause of urinary tract infection: qualitative interview study. BM/ 2010:340:c279. doi:10.1136/bmj.c279.

37 Knottnerus BJ, Geerlings SE, Moll van Charante EP, ter Riet G. Women with symptoms of uncomplicated urinary tract infection are often willing to delay antibiotic treatment: a prospective cohort study. BMC Fam Pract 2013;14:71. doi:10.1186/1471-2296-14-71.

38 Little P, Moore MV, Turner S, et al. Effectiveness of five different approaches in management of urinary tract infection: randomised controlled trial. BM/2010·340:c199 doi:10.1136/bmj.c199.

39 Brown KA, Khanafer N, Daneman N, Fisman DN. Meta-analysis of antibiotics and the risk of community-associated Clostridium difficile infection. Antimicrob Agents Chemother 2013;57:2326-32. doi:10.1128/AAC.02176-12.

40 Deshpande A, Pasupuleti V, Thota P, et al. Community-associated Clostridium difficile infection and antibiotics: a meta-analysis. J Antimicrob Chemother 2013;68:1951-61. doi:10.1093/jac/dkt129.

Supplementary information: tables S1-S3 